\title{
Frozen shoulder: prospective clinical study with an evaluation of three treatment regimens
}

\author{
D. Y. BULGEN, A. I. BINDER, B. L. HAZLEMAN, \\ J. DUTTON, AND S. ROBERTS \\ From the Department of Rheumatology Research, Addenbrooke's Hospital, Hills Road, \\ Cambridge CB2 $2 Q Q$
}

SUMmARY Forty-two patients with frozen shoulder were followed up closely for eight months. They were all taught pendular exercises and randomly allocated to one of four treatment groups: (a) intraarticular steroids, (b) mobilisations, $(c)$ ice therapy, $(d)$ no treatment. This study has shown that there is little long-term advantage in any of the treatment regimens but that steroid injections may benefit pain and range of movement in the early stages of the condition.

Frozen shoulder is a rheumatological enigma. Precise definition varies, response to the many recommended treatments is often capricious, and many of the published reports are difficult to interpret because they are based on small series or contain inadequate clinical or treatment details.

A wide variety of treatments have been investigated, including local ${ }^{12}$ or oral $^{3}$ steroids, manipulation under anaesthesia (MUA), ${ }^{45}$ stellate ganglion block, ${ }^{6}$ physiotherapy,${ }^{7}$ infiltration brisement, ${ }^{8}$ and radiotherapy. ${ }^{9}$ There is no general agreement in favour of one form of treatment, and the response to a particular treatment varies in different series. The value of local steroid injections may be taken as an example. In a retrospective study Hazleman ${ }^{10}$ could not show any difference between local steroid injection, MUA, and physiotherapy. Similarly, but in a prospective study, Lee et al. ${ }^{11}$ found no significant advantage of local steroid injection plus physiotherapy against heat plus physiotherapy. However, Roy and Oldham ${ }^{12}$ have reported local steroid injections to be highly effective in patients with painful, generally restricted shoulders. Quin ${ }^{2}$ also found steroid injections to be effective, but he noted that the improvement in pain and range of movement was short-lived. Treatment regimens should be judged against natural recovery, which is often slow and incomplete. Simmonds ${ }^{13}$ studied 21 patients for more than three years. Nine had persistent weakness and pain, six had weakness or

Accepted for publication 19 November 1983. Correspondence to Dr B. L. Hazleman. loss of movement, and only six regained normal function.

Whether any clinical features such as mode of onset can predict or influence the eventual outcome also remains unclear. Hazleman ${ }^{10}$ found early presentation but not severity or type of onset (spontaneous or traumatic) to influence the recovery time. Clarke et al $^{14}$ reported a trend for young males and dominant arm involvement to be associated with a less favourable prognosis. As both the studies were performed retrospectively, interpretation of these trends is difficult. Fearnley and Vadasz ${ }^{15}$ noted that patients with a raised sedimentation rate responded better to steroid injections. However, they were unable to show that the duration of symptoms at presentation or the mode of onset affected the prognosis.

Our aim was therefore, firstly, to study a carefully defined patient group and assess three treatment regimens against non-treatment over a long period.

\section{Patients and methods}

Forty-five patients entered the study, but three were subsequently withdrawn: one developed very acute pain after an arthrogram, one obtained physiotherapy elsewhere, and one failed to attend. Forty-two patients, 28 females and 14 males, with previously untreated frozen shoulder completed this study. Criteria for inclusion were pain in the shoulder for at least one month, with sleep disturbance due to night pain and inability to lie on the affected shoulder. All active and passive shoulder movements were res- 
tricted, with a reduction in external rotation of at least $50 \%$. Patients with any sensory symptoms or signs in the affected arm or radiation of pain to the neck were excluded. Patients with generalised arthritis, fractures, or dislocations of the humerus, cervical spondylosis, or evidence of referred pain were also excluded.

Twenty-two involved the dominant arm and only one became bilateral. The onset was spontaneous in 27 cases and followed minor trauma in 14 patients. Twelve of the patients were manual workers, but this did not influence the arm involved. Although the frozen shoulder followed a herpes zoster infection of the T8 dermatone in one patient, no other known precipitating factors such as strokes, myocardial infarctions, or diabetes mellitus were present.

The age range was 44-74 years (mean 55.8), and the duration of the condition at presentation was 1-12 months (mean 4.8). Fig. 1 shows the distribution of age, sex, and disease duration before treatment.

Clinical assessment was performed before treatment, weekly for 6 weeks and monthly for a further 6 months by an independent observer who was not aware of the treatment given.

Pain scores were recorded on $10 \mathrm{~cm}$ visual analogue scales for night pain, pain on movement, and rest pain during the day. Patients were also asked to state whether the various types of pain were better, the same, or worse.

The range of shoulder movements was measured according to the method of Clarke et al. ${ }^{16}$ The hydrogoniometer produced by Geigy was strengthened and fitted with Velcro straps as they described. Only passive movements were recorded. Clarke et al. measured external and total rotation

Duration less than $3 / 12$ Duration more than $3 / 12$
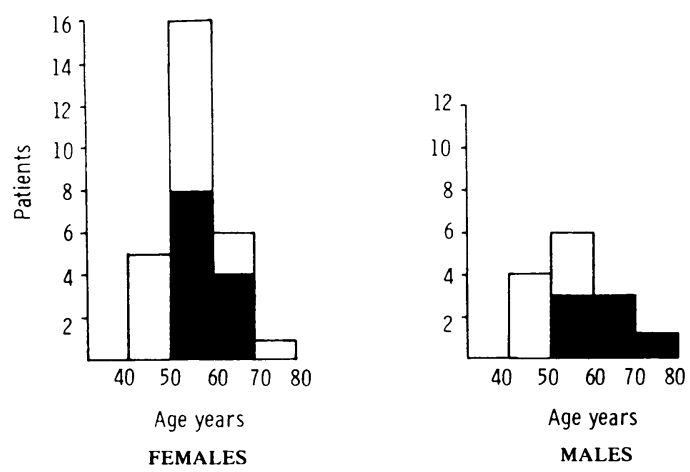

Fig. 1 Distribution of patients according to age, sex and disease duration at presentation. and glenohumeral abduction. We used the same method to measure also total flexion and abduction and glenohumeral flexion with the goniometer attached to the upper arm and the patient seated. To assess glenohumeral range the shoulder girdle was stabilised by firm downward pressure over the acromion process. Internal rotation was recorded as the vertebral spine reached by the extended thumb. Clarke et al. recorded measurements to the nearest $2^{\circ}$. We found it more reproducible to measure to the nearest $5^{\circ}$. The measurements were performed twice, and if they differed by more than $5^{\circ}$ they were repeated once more and the mean of the closest two observations was taken. Measurements were always taken before and not after a physiotherapy session. Similar measurements were taken of both shoulders of 42 age- and sex-matched healthy controls and 36 other controls of a similar age group to provide a normal population sample.

\section{TREATMEN T}

The patients were randomly allocated to one of four treatment groups.

(1) Steroid group (11 patients). Methyl prednisolone acetate $20 \mathrm{mg}$ and $1 \%$ lignocaine hydrochloride $0.5 \mathrm{ml}$ were injected into the subacromial bursa and a similar amount into the shoulder joint by the anterior route, weekly, for three weeks.

(2) Mobilisation group (11 patients). Maitland's mobilisations ${ }^{17}$ were given by a research physiotherapist to these patients, three times weekly for six weeks.

(3) Ice group (12 patients). Ice packs followed by proprioceptive neuromuscular facilitation (PNF) was supervised by the same research physiotherapist with a similar time schedule to the mobilisation group.

(4) Non-treatment group (8 patients).

All patients were taught pendular exercises and advised to do them for 2-3 minutes every hour. Non-salicylate analgesics and diazepam $5 \mathrm{mg}$ at night were available as required.

\section{Results}

CLIN I CA L

Thirty-nine patients reported excellent general health, one had a chronic duodenal ulcer, another had hyperventilation syndrome, and one woman was hypertensive with a history of depression. Five patients had previously suffered from a painful stiff shoulder on the opposite side. Despite their age they were a very fit group of patients with little past illness.

The patients' occupations were as follows: 12 were engaged in heavy manual labour and 8 light 
manual labour, 15 were housewives, and the other 7 had sedentary occupations. There was no association between heavy manual work and dominance of the frozen shoulder. Three patients were unable to work because of the frozen shoulder. They included a carpenter, a nurse, and a hairdresser. A railway worker found that physiotherapy (ice) exacerbated his pain and was then off work for two months. All other patients remained at work.

The initial drug treatment prescribed by general practitioners is shown in Fig. 2. It can be seen that half the patients were given a non-steroidal antiinflammatory drug NSAID but only $3 / 21$ patients found the drug helpful. An analgesic was found helpful by $5 / 10$ patients. Ten patients were not prescribed any drug therapy. By the time the patient came to clinic the drug therapy had changed (Fig. 3). Only six patients were taking NSAIDs, 19 had become disillusioned and were not taking anything, and three patients were taking simple analgesics.

During the study 12 patients did not take any drugs, 26 took simple analgesics for varying lengths of time, but mainly during the first six weeks. Eight patients with severe night pain were prescribed diazepam $5 \mathrm{mg}$ at night, and all reported some benefit. Again, they mainly took it during the first six weeks. At the end of the study one patient was still taking an occasional Distalgesic tablet (dextropropoxyphene and paracetamol) and one patient required diazepam $2 \mathrm{mg}$ b.d. and $5 \mathrm{mg}$ nocte plus Paramol-118 (paracetamol) tablets 2 nocte. So far

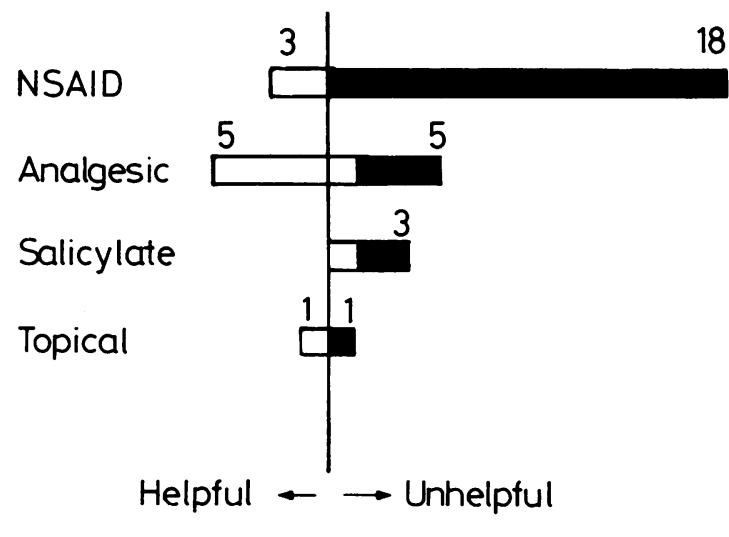

Therapy stopped

Therapy cont

Fig. 2 Initial drug treatment prescribed by general practitioners $(N S A I D=$ non-steroidal anti-inflammatory drug).

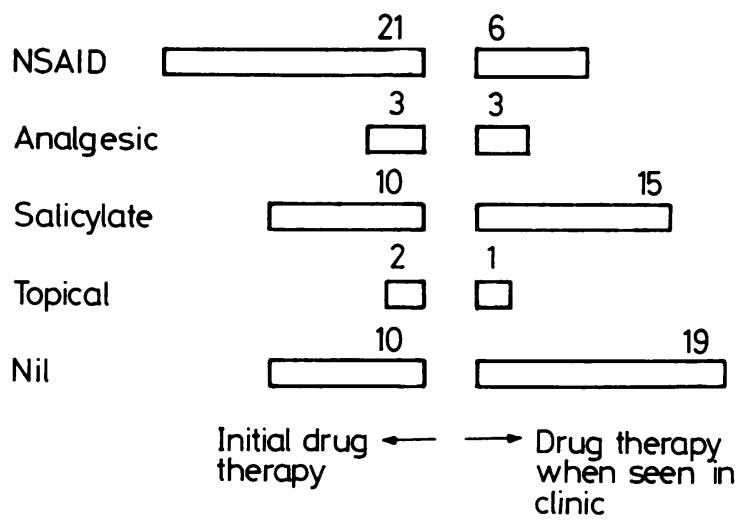

Fig. 3 Drug treatment at outpatient appointment. (NSAID=non-steroidal anti-inflammatory drug)

as initial physical advice by general practitioners was concerned, none was given to 28 patients, 2 were told to rest the shoulder, 6 were told to exercise it through pain and the remaining six were advised to use the shoulder normally but to avoid strain.

On examination all patients had very limited shoulder movements: mean total flexion $99^{\circ}$ (range $50^{\circ}-135^{\circ}$ ), mean glenohumeral flexion $51^{\circ}$ (range $40^{\circ}-70^{\circ}$ ), mean total abduction $69^{\circ}$ (range $20^{\circ}-135^{\circ}$ ), mean glenohumeral abduction $40^{\circ}$ (range $0^{\circ}-70^{\circ}$ ), mean total external rotation $13^{\circ}\left(\right.$ range $\left.0^{\circ}-35^{\circ}\right)$, and mean total rotation $41^{\circ}$ (range $\left.0^{\circ}-100^{\circ}\right)$. Sixteen patients were found to have pain on resisted movements, and a further nine patients had local tenderness mainly over the tuberosities, but they appeared to be no different from the other patients so far as the pattern of recovery was concerned. Half of the patients presented within three months of the onset of the disease and the rest later (Fig. 1). The patients seen early complained of slightly more pain at rest and at night than the other patients, but the difference did not reach statistical significance.

Initial range of movement was similar in both groups.

\section{PAIN MEASUREMENT}

It was noted that many patients found difficulty in transcribing their verbal descriptions of pain to the visual analogue scales, so that by the end of the study we felt that little reliance could be placed on any detailed analysis of these scales. However, verbal reports of progress seemed more consistent and reliable.

All patients reported an improvement in pain during the study, but 17 still had residual pain at the end of it, usually mild, but moderate in four. Residual pain was commoner in the ice group, 
affecting half the patients, but was equally distributed in the other groups. All types of pain were still experienced-rest pain, night pain, and pain on movement. The maximum improvement in pain was achieved by the fourth week of treatment and then continued slowly after that. This was most obvious in the steroid group, but did not reach statistical significance.

\section{SHOULDER MEASUREMENT}

Recovery curves were plotted for each movement showing the average improvement in degrees between consecutive visits in each treatment group against time (Figs. 4-7). These were further analysed by the method described by Lee et al. ${ }^{11}$
Component analysis was used to compute a principal component, C, which accounted for $59 \%$ of the total variation between the patients initially. This was equal to: $\mathrm{C}=(0.536 \times$ total flexion $)+(0.201 \times$ glenohumeral flexion $)+(0.679 \times$ total abduction $)$ $+(0.263$ glenohumeral abduction $)+(0.079 \times$ external rotation $)+(0.369 \times$ total rotation $)-$ $137 \cdot 7$.

The component $\mathrm{C}$ was calculated for each patient at each time, and the cumulative effect was plotted as for the individual measurements. It can be seen that a low value for $\mathrm{C}$ represented a poor range of movement and a higher value an improvement. Thus we were able to draw a cumulative recovery curve (Fig. 8).

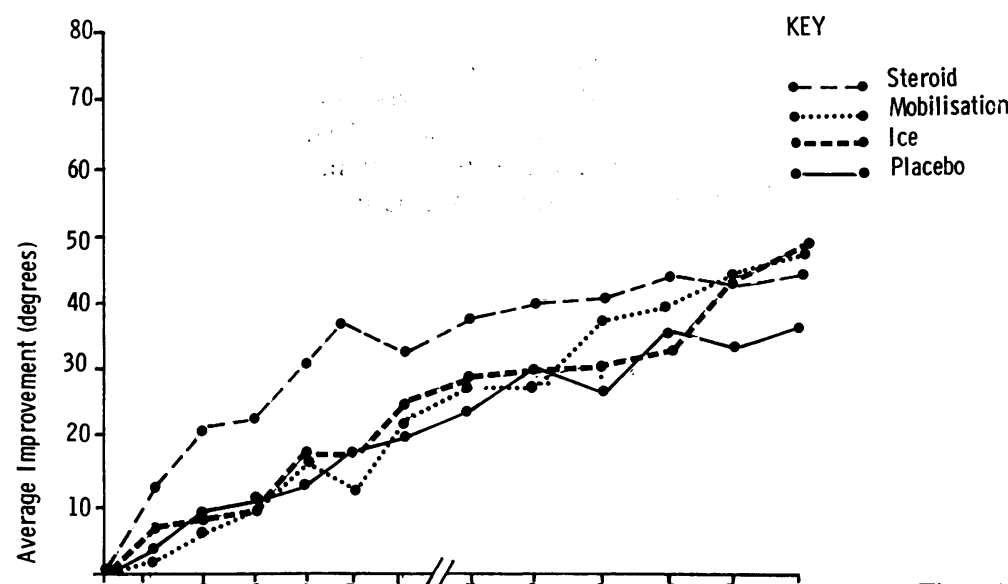

Figs. 4-7 Recovery curves showing average improvement in degrees between consecutive visits for total flexion (Fig. 4), total abduction (Fig. 5), external rotation (Fig. 6), and total rotation (Fig. 7).

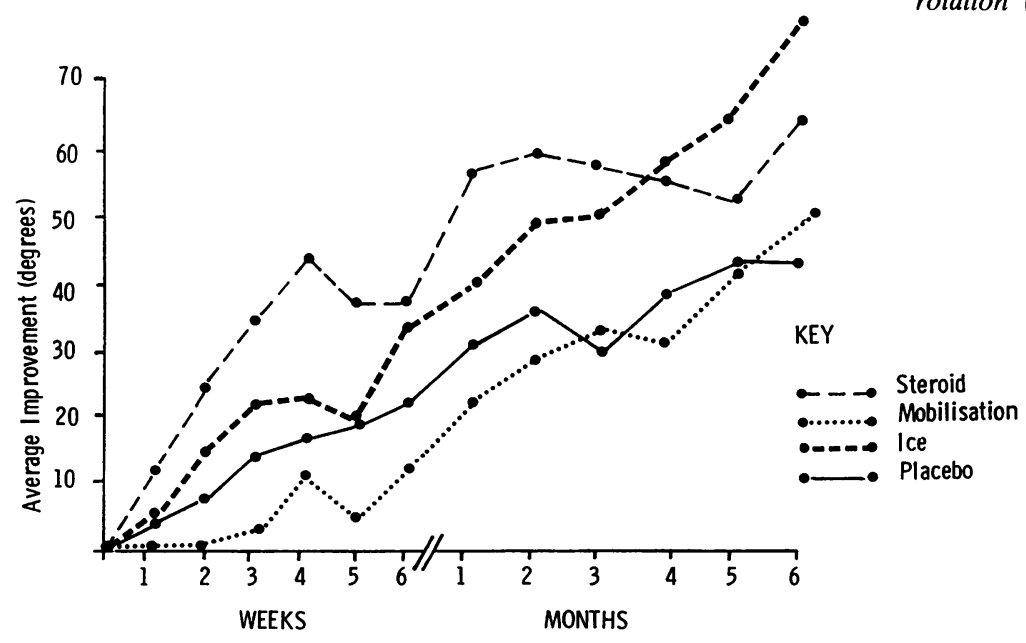

Fig. 5 
Frozen shoulder: prospective clinical study with an evaluation of three treatment regimens 357

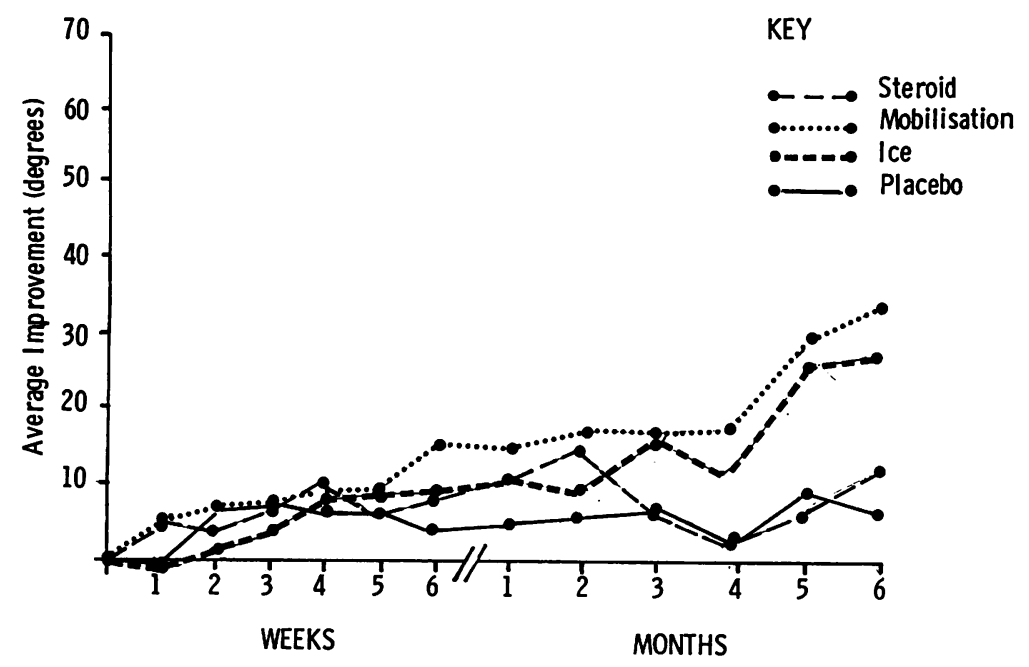

Fig. 6

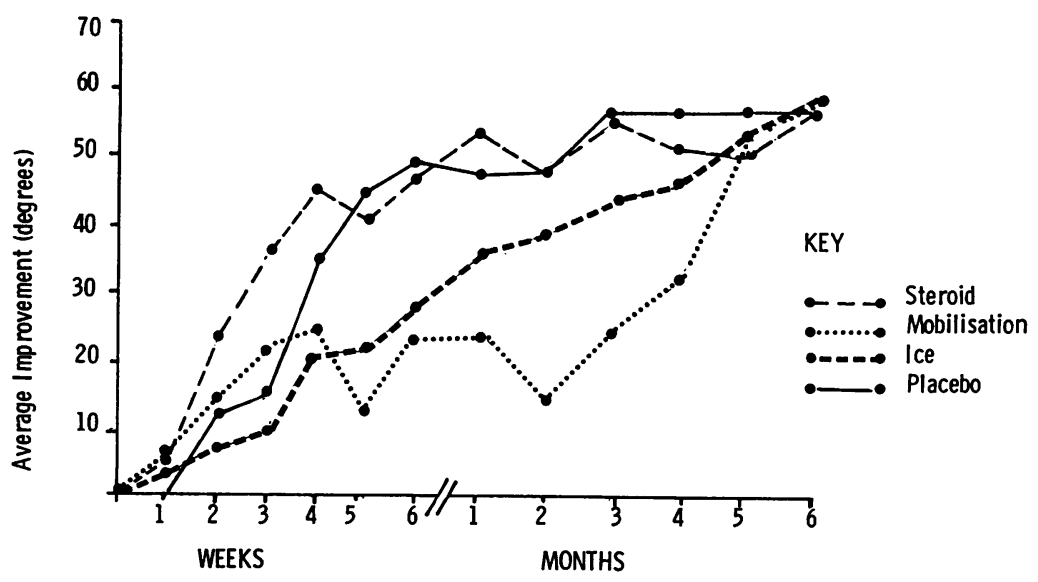

Fig. 7

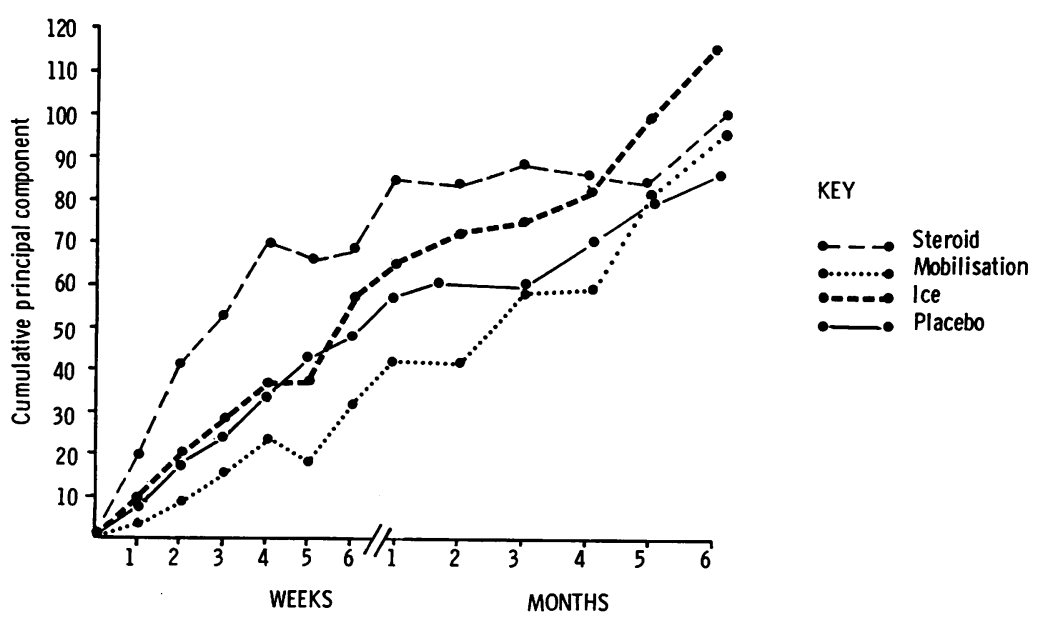

Fig. 8 Improvement in cumulative principal component. 


\section{TREATMEN T}

From the graphs (Figs. 4-8) it can be seen that the initial improvement in movement was most marked in the steroid group, but by the end of the study the groups were similar. This was confirmed by performing a one-way analysis of variance on the principal component figures. At six weeks-i.e., the end of treatment-the groups were significantly different at the $2 \%$ level, but by the end of the study there was no significant difference between the groups.

From the graphs it can be seen that the major improvement in range occurs within the first four weeks, suggesting that a course of treatment should be limited to this length of time and that local steroid injections should be the initial treatment of choice.

\section{OTHER FACTORS}

The principal component $\mathrm{C}$ was also used to assess the effect of other factors on the rate of recovery of range as long as these factors were equally distributed in the treatment groups. Thus it was found by an unpaired $t$ test at six weeks and at the end of the study that there was no significant difference in the

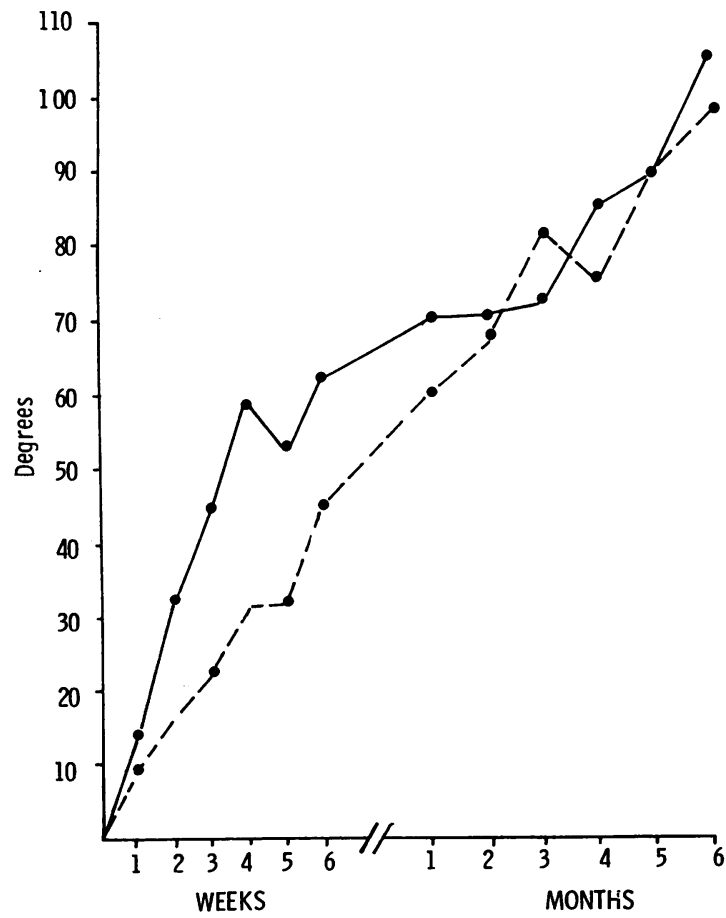

Fig. 9 Effect of early and late presentation on recovery of range. Presentation at three months or earlier at later than three months rate of recovery of shoulder movement whether the dominant or the non-dominant shoulder was affected. Similarly, presentation before or after three months had no statistically significant effect on the recovery of shoulder movement at six weeks and at the end of the study, although from the graph (Fig. 9) it appears that the early presentation group had a better initial response. There was also no relationship between the principal component at the start of the study and at the end. Thus the initial severity of restriction gave no indication of the final outcome as far as range was concerned.

\section{CONTROL MEASUREMENTS}

The range of shoulder movement in our controls was compared for right and left shoulders and for dominant and non-dominant shoulders by Student's $t$ test (Table 1). No significant difference was found $(p<0 \cdot 05)$, but rotation and particularly flexion were better on the left or non-dominant shoulder and abduction was better on the right or dominant shoulder. Only 12 controls were left dominant, so that the above comparisons could be expected to give very similar results.

Using the right shoulder of the 76 controls and ignoring age, we compared the range of movement between males and females and found a significantly greater range in females, for total abduction $(p<0.02)$ total rotation $(p<0.02)$ and external rotation $(\mathrm{p}<0.001)$ (unpaired $t$ test with $48 \mathrm{DF})$. Total flexion was not significantly different between the sexes. Performing a linear regression against age gave significant decreases $(\mathrm{p}<0.001)$ in the range of all movements except external rotation. External rotation seemed to be poor in the youngest patients, under 50 years old. In view of the above findings a comparison between the same four measurements in our patients at 6 months after treatment was made

Table 1 Control shoulder measurement. Comparison of right versus left shoulder and dominant versus non-dominant shoulder

\begin{tabular}{lrrl}
\hline & Mean & \multicolumn{1}{l}{$t_{75}$} & $p r>t_{75}$ \\
\hline Comparison of right $v$. left shoulders & & & \\
Total flexion (R-L) & -0.7 & -0.77 & 0.45 \\
Total abduction & $0 \cdot 5$ & $0 \cdot 53$ & $0 \cdot 60$ \\
External rotation & -0.9 & $-1 \cdot 77$ & 0.08 \\
Total rotation & -1.4 & $-1 \cdot 15$ & $0 \cdot 26$ \\
& & & \\
Comparison of dominant $v$. & & & \\
non-dominant shoulders & & & \\
Total flexion (Dom-non-dom) & -0.9 & -0.99 & 0.33 \\
Total abduction & 0.7 & 0.75 & 0.46 \\
External rotation & -0.9 & $-1 \cdot 77$ & 0.08 \\
Total rotation & -0.2 & -0.16 & 0.87 \\
\hline
\end{tabular}

$R=$ right. $L=$ left. Dom $=$ dominant 
Table 2 Comparison between patients' range (post-treatment) against age and sex-matched controls

\begin{tabular}{ll}
\hline & Mean decrease (degrees) \\
\hline Total flexion & $27 \cdot 8$ \\
Total abduction & $22 \cdot 5$ \\
External rotation & 22.9 \\
Total rotation & $63 \cdot 8$
\end{tabular}

Three comparisons were made, using controls' $R$ and $L$, and same R/L as patients' affected shoulder, and the least values recorded.

with age- and sex-matched controls, and all four measurements were significantly lower in the patients $(p<0.0001)$, whether the controls' right or left or same right or left, as the patients' affected shoulders were used (Student's $t$ test). Table 2 shows the least reduction in range in the three comparisons made in our patients compared with the controls. Only four of the $42(10 \%)$ patients had attained a range comparable with the minimum range of all the controls of similar age (assessed in each decade from 40 to 70 years) and sex. Therefore six months after completion of treatment our patients still had a significant deficit in the range of shoulder movement, though the majority were pain free.

\section{Discussion}

It had been hoped that this study would identify clinical subgroups in the frozen shoulder syndrome distinguished by clinical factors, immunological ${ }^{18}$, arthrographic, or technetium scan features ${ }^{19}$ which would predict perhaps the likely rate of recovery or response to a specific treatment. Unfortunately this has not happened.

In our study the spontaneous onset of frozen shoulder, although more common, could not be differentiated from the cases with preceding trauma by the severity of the initial pain, restriction in the range of movements, or the rate of recovery.

Hazleman, ${ }^{10}$ and later Oldham, ${ }^{12}$ have suggested that early presentation is associated with a shorter total duration. The patients whom we saw early tended to improve rapidly, but this was not sustained, and there did not appear to be any long-term benefit (Fig. 9).

We have found less benefit from physiotherapy compared with non-treatment than Lee et al, ${ }^{11}$ but they used a slightly different patient group in that $16 / 25$ patients had had a frozen shoulder for more than one year, some for five years or more. Their placebo group was measured after six weeks, while the active treatment groups were measured weekly.
We saw all our patients weekly and gave them similar encouragement in order to minimise differences between them.

We found the method of measurement recommended by Clarke et al. ${ }^{14}$ reproducible and easy to do. We agree with Hazleman's ${ }^{10}$ finding that patients often claimed that they had regained full movement, but objective testing showed that this was not so. Clarke et al. ${ }^{14}$ reported that recovery was better when the non-dominant shoulder was involved. We found the opposite to be true, but statistical significance was not reached in either study. He also noted that $42 \%$ of his patients had significant limitation of shoulder movement after six years. $90 \%$ of our patients had not attained a range comparable with the minimum range of a control group of similar age and sex by 6 months follow-up, $17 \%$ still having severe restriction.

Our control shoulder measurements confirmed the finding of Allander et al. ${ }^{20}$ and Clarke et al. ${ }^{16}$ that females have a greater shoulder range than males but that there is no difference between right and left shoulders or dominance.

In their study of the management of the painful shoulder Roy and Oldham ${ }^{12}$ included a group of 55 patients with painful limitation of all shoulder movement and pain at night, similar to the patients in our study. They reported an excellent response to paired steroid injections, and so we used similar steroid dosage, frequency, and method of injection to try to reproduce their results. The age range and the duration of the frozen shoulder were similar in both groups. The only obvious differences were that we used a smaller volume of lignocaine, injected the shoulder joint from the anterior route, and the shoulders of our patients were probably more restricted. $95 \%$ of Roy and Oldham's patients were pain free eight weeks after they were first seen, having had two or three pairs of injections, but only four out of $11(36 \%)$ of our patients given steroid injections were pain-free at that stage. Similarly, we achieved less improvement in the range of movement in our patients. The average pretreatment total abduction was $72 \%$ and $60.5 \%$ respectively. $73 \%$ or $44 / 60$, shoulders in their series had achieved $150^{\circ}$ or more within eight weeks of starting treatment, but only $18 \%$ or $2 / 11$, of our patients had achieved this after 10 weeks.

Obviously our steroid treatment results are much less satisfactory than those of Roy et al. ${ }^{21}$, perhaps reflecting a different type of patient and different measuring techniques, but we do agree with their finding that such an injection regimen is helpful. They stress the effectiveness of paired injections into the subacromial bursa and the shoulder joint over injections into just one site, which may partly 
explain some of the differences in our results compared with those of the study by Lee et al. ${ }^{11}$

This study has shown that there is little long-term advantage in any of our treatment regimens over no treatment, but that steroid injections may benefit pain and range of movement in the early stages. There appears to be little place for physiotherapy alone, and, if used, it should not be continued for more than four weeks.

Significant range restriction, often unrecognised by the patient, was confirmed in most of our patients six months after treatment. Further follow-up was therefore undertaken to determine the long-term outcome.

We thank the Arthritis and Rheumatism Council for support.

\section{References}

1 Lloyd Roberts G C, French P R. Periarthritis of the shoulder. Br Med J 1959; i: 1569-72.

2 Quin C E. Frozen shoulder: evaluation of treatment with hydrocortisone injections and exercises. Ann Phys Med 1965; 8: 22-9.

3 Blockley N J, Wright J K, Kellgren J H. Oral cortisone therapy in periarthritis of the shoulder. Br Med J 1954; i: 1455-7.

4 Bloch J, Fischer F K. Frozen shoulder. Acta Rheumatol (Geigy) 1961; no.15.

5 Thomas D, Williams R A. Smith D S. The frozen shoulder: a review of manipulative treatment. Rheumatol Rehabil 1980; 19: $173-9$.

6 Williams N E, Seifert M H, Cuddigan J H P, Wise R A. Treatment of capsulitis of the shoulder. Rheumatol Rehabil 1975; 14: 236.

7 Mattingly S. Pain in the shoulder. Ann Phys Med 1960; 5: 267-81.
8 Simon W H. Soft tissue disorders of the shoulder: frozen shoulder, calcific tendinitis and bicipital tendinitis. Orthop Clin North Am 1975; 6: 521-39.

9 Quin C E. Humeroscapular periarthritis: observations on the effect of $x$-ray therapy and ultrasonic therapy in cases of 'frozen shoulder'. Ann Phys Med 1969; 10: 64-9.

10 Hazleman B L. The painful stiff shoulder, Rheumatol Rehabil 1972; 11: 413-21.

11 Lee P N, Lee M, Haq A M M M, Longton E B, Wright V. Periarthritis of the shoulder-trial of treatments investigated by multivariate analysis. Ann Rheum Dis 1974; 33: 116-9.

12 Roy S, Oldham R. Management of painful shoulder. Lancet 1976; ii: $1322-4$.

13 Simmonds F A. Shoulder pain with particular reference to the 'frozen' shoulder. J Bone Joint Surg 1949; 31B: 426-32.

14 Clarke G R, Willis L A, Fish W W, Nichols P J R. Preliminary studies in measuring range of motion in normal and painful stiff shoulders. Rheumatol Rehabil 1975; 14: 39-46.

15 Fearnley M E, Vadasz I. Factors influencing the response of lesions of the rotator cuff of the shoulder to local steroid injection. Ann Phys Med 1969; 10: 53-63.

16 Clarke G R, Willis L A, Fish W W, Nichols P J R. Assessment of movement at the gleno-humeral joint. Orthopaedics (Oxford) 1974; 7: 55-71.

17 Maitland G D. Peripheral manipulations. Butterworth: London, 1970: 25-51.

18 Bulgen D Y, Binder A I, Hazleman B L, Park J R. Immunological studies in frozen shoulders. J Rheumatol 1983; 9: 893-8.

19 Binder A I, Bulgen D Y, Hazleman B L, Tudor J, Wraight P. Frozen shoulder. 3. An arthrographic and radionuclear scan assessment of the frozen shoulder. Ann Rheum Dis (same issue).

20 Allander E, Bjornsson O J, Olafsson O, Sigfusson N, Thorsteinsson J. Normal range of joint movements in shoulder, hip, wrist and thumb with special reference to side: a comparison between two populations. Int J Epidemiol 1974; 3: 253-61.

21 Roy S, Oldham R, Nichol F E. Frozen shoulder: adhesive capsulitis. Br Med J 1982; 284: 117-8. 